\title{
Euthanasia and palliative sedation in Belgium
}

Raphael Cohen-Almagorand E Wesley Ely

This is the accepted version of an article published in BMJ Supportive and Palliative Care (Online First: January 2018).

\section{Abstract}

The aim of this article is to use data from Belgium to analyse distinctions between palliative sedation and euthanasia. There is a need to reduce confusion and improve communication related to patient management at the end of life specifically regarding the rapidly expanding area of patient care that incorporates a spectrum of nuanced yet overlapping terms such as palliative care, sedation, palliative sedation, continued sedation, continued sedation until death, terminal sedation, voluntary euthanasia and involuntary euthanasia. Some physicians and nurses mistakenly think that relieving suffering at the end of life by heavily sedating patients is a form of euthanasia, when indeed it is merely responding to the ordinary and proportionate needs of the patient. Concerns are raised about abuse in the form of deliberate involuntary euthanasia, obfuscation and disregard for the processes sustaining the management of refractory suffering at the end of life. Some suggestions designed to improve patient management and prevent potential abuse are offered.

The published version is available at http://dx.doi.org/10.1136/bmjspcare-2017-001398

(C) Authors (or their employer(s)) 2018 


\section{Introduction}

The central premise of this manuscript is that the appropriate and measured use of sedation at the end of life to help reduce human suffering can transition into confusing and problematic care because of three main reasons. First, physicians understand different practicesas being complicit with 'euthanasia'. This confusion in terminology hampers effective monitoring and control over the practice of euthanasia. ${ }^{-}$-Second, there is ambiguity and uncertainty regarding physicians' intentions in administrating sedation. There is a grey area between sedation intended only to relieve suffering versus sedation deliberately intended to hasten death. $\frac{2-4}{4}$ Third, as a result of the above problems, the use of sedation is opened to abuse. Abuse of sedation occurs when clinicians sedate patients at the end of their lives with the primary goal of hastening and causing the patient's death. ${ }^{5-z}$ Continuous deep sedation until death might be initiated without consent or request of either the patient or the family. One study showed that in the case of 1200 patients in Belgium, the intention of the medical team was explicitly to shorten life. ${ }^{8}$ This is inappropriate use of sedation. Explicit life-shortening intentions are reported by physicians. ${ }^{9-11}$ Administration of medication with the covert intention to hasten death is ethically wrong. ${ }^{512-16}$

This article clarifies the terminology and explains why palliative sedation is not euthanasia (as euthanasia rests on intent to end life), and why it is important to be as precise as possible in applying the right terminology in describing different practices at the end of life. We use data from Belgium to present a discussion that will help others avoid communication and end-of-life management pitfalls in this confusing area of medical practice. It is argued that in too many instances, Belgian physicians are obfuscating palliative sedation in order to euthanise patients with their consent or to deliberately hasten patient deaths often without any process of consent. Finally, we make some constructive suggestions aimed at serving the patients' best interests, preventing abuse and improving decision-making processes at the end of life.

\section{The Belgian Euthanasia Act and sedation}

Euthanasia is defined as the act, performed by a third party, which intentionally ends the life of a person at his or her explicit request. The legislation lays out the terms for doctors to end the lives of patients who are in a state which, medically, is without prospect of improvement. ${ }^{17}$ Euthanasia requests are approved only if the patient is in a hopeless medical condition and complains of constant and unbearable physical or mental pain which cannot be relieved and is the result of a serious and incurable accidental or pathological condition. At least 1 month must elapse between the written request and the mercy killing. ${ }^{18}$ The 1 month-stipulation is required only when the patient is not 'terminally ill' (eg, quadriplegia). Since the enactment of the Euthanasia Act in 2002, reports are being published by the Belgian 
Federal Control and Evaluation Commission, established by the government in September 2002, assigned to monitor the law's application. A constant increase in registered euthanasia cases has been observed, predominantly in the Flemish (the Dutch-Flemish speaking) part of Belgium. ${ }^{19-21}$ Johan Bilsen et al found that the enactment of the Euthanasia Act in Belgium was followed by an increase in almost all types of medical end-of-life practices: euthanasia, intensified alleviation of pain, withholding or withdrawing life-prolonging treatment, and continuous and deep sedation until death. $\stackrel{2022}{\mathrm{i}}$.

Many Belgian scholars tend to cluster together practices under broad categories such as End-of-Life Decisions $\underline{123}$ and Life-Ending Acts Without Explicit Request. ${ }^{24} 25$ This clustering creates a false continuum between palliative care and euthanasia. Furthermore, Belgian researchers describe the practice of slowly increasing the dosage of sedatives while gradually withdrawing treatment in different terms: continuous sedation, ${ }^{26}{ }_{27}$ continued sedation to unconsciousness, ${ }^{28}$ eontinuous deep sedation, ${ }^{29}$ continuous sedation at the end of life, ${ }^{26-2730}$ continuous sedation until death, ${ }^{31}$ continuous deep sedation until death, 9 palliative sedation and terminal sedation. $\frac{2832}{1}$ ii These terms are often used interchangeably, which adds to the confusion.

\section{Clarifications}

\section{Palliative care}

This refers to the active, comprehensive and total care of patients whose disease is not responsive to curative treatment, where maintaining the control of pain and other sources of suffering (eg, anxiety, psychological, social and spiritual problems) is paramount. Palliative care is action by the medical community to optimise the comfort, function, relationships, healing, dignity and preparation for the death of the person, who has life-threatening illness, and their family. ${ }_{33}^{334}$

\section{Palliative sedation}

This is the use of sedative medication to relieve intolerable suffering during a patient's active dying process by a reduction of the patient's level of consciousness. ${ }^{35-37}$ To be clear, euthanasia involves administering deliberately lethal doses of barbiturates and paralytic agents. In contrast, palliative sedation involves administration of potent psychoactive medications such as opioids, antipsychotics, benzodiazepines or propofol (intravenous, oral or subcutaneous) in a manner proportionate to prevailing symptoms to accomplish relief of suffering with mild, moderate or deep sedation. 38390 pioids are used for alleviating pain and/or refractory dyspnoea. Shortness of breath causes great suffering and opioids can help patients; thus palliation with opioids is appropriate up to a point. If used to help palliate suffering, this is ethically sound. If consciously used and intentionally used to stop breathing entirely and kill the patient, this is unethical. At any rate, shortening life is not the explicit or implicit intent of palliative sedation.

A significant minority of dying people (cancer research indicates between a tenth and a quarter) experience refractory symptoms such as fatigue, dyspnoea, pain, 
anxiety and nausea, or extreme distress unresponsive to conventional therapies. In such circumstances, sedation may be used to decrease or remove consciousness. ${ }^{28}$ According to members of the Department of Intensive Care, Erasme Hospital in Brussels, sedation is encouraged to keep patients calm, collaborative and comfortable. Patients with severe respiratory failure received sedatives for the longest duration, followed by postoperative and haemodynamically unstable patients. 40

While euthanasia shortens life, palliative sedation does not shorten life. ${ }^{41}$ Maltoni et al argued that palliative sedation, when appropriately indicated and correctly used to relieve unbearable suffering, does not have any detrimental effect on survival of patients with terminal cancer. 2243 Palliative sedation may even prolong life to some extent because it prevents exhaustion as a result of suffering. 44 In Temel's landmark trial of patients with metastatic non-small cell lung cancer, as compared with usual care, those randomised to early palliative care led to significant improvements in both quality of life and mood with less aggressive care at the end of life yet longer survival. 45

\section{Continuous sedation}

Anquinet et al define continuous sedation as the practice where sedation, that is, the lowering of the patient's consciousness, is administered continuously until the time of death. The authors explain that this practice can only be performed when the patient has a very limited life expectancy and that it does not aim at the death of the patient. ${ }^{46}$ This definition lumps together continuous sedation and continuous sedation until death. Other scholars use the short and imprecise acronym CS

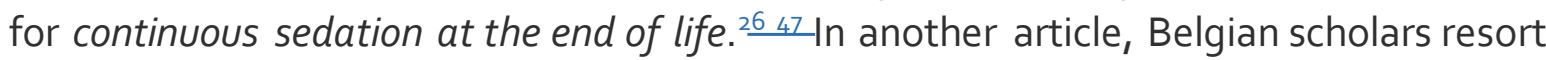
to yet another term, continuous deep sedation (CDS) to describe the same practice. ${ }^{29}$ Some of these scholars, together with others, use another acronym, CSD, to describe the same phenomenon, this time calling it continuous sedation until death. ${ }^{31}$ In his comments on this article, Kasper Raus explained that 'continuous sedation' is the broad concept that includes both light and deep sedation while 'continuous deep sedation' is a particular form of continuous sedation, namely that form where the patient is deeply sedated. But this is not clear in the article. It is also unclear whether the 'continuous deep sedation' patient group is different (smaller?) from the 'continuous sedation' patient group.

\section{Terminal sedation}

The term terminal sedation, which we believe is confusing regarding clinical intent, was introduced by Enck in a 1991 review article published in the American Journal of Hospice and Palliative Care. ${ }^{4}$ Since then, terminal sedation has been a widely used term, although the term plays only a marginal role in most other international clinical studies. ${ }^{49}$ Chater et al examined the response of 61 selected palliative care experts to a proposed definition for terminal sedation. The experts opined that the term 'terminal sedation' should be abandoned and be replaced with another such as 
palliative sedation. ${ }^{5}$ Several other authors object to the use of the term terminal sedation. $\frac{51-53}{2}$

The practice of sedating patients until death, while is clinically appropriate in some patients, is significantly more prevalent in Belgium than euthanasia. A study among six European countries (Belgium, Denmark, Italy, The Netherlands, Sweden and Switzerland) with comparative epidemiology of terminal disease showed that Belgium had the second highest percentage of continuous deep sedation, ${ }^{54}$ also termed continuous and deep sedation until death ${ }^{20.55: 8.2 \%}$ of all deaths. The report on continuous deep sedation until death conducted in Flanders in 2007 showed that $14.5 \%$ of all deaths occurred while using this practice. This is a significant increase compared with $8.2 \%$, just 6 years earlier. ${ }^{20} 5657$

One definite downside of such deep sedation is that it usually results in a fairly profound drug-induced delirium, which is certainly a form of suffering that many describe as torturous. The hazard of such a drug-induced delirium is that profound cognitive dysfunction deprives some otherwise competent and lucid patients of very meaningful and desired periods of communication with loved ones, spiritually focused interactions and relationship reconciliation. Drug-induced delirium is often idiosyncratic in nature and there is a great degree of interindividual variability with regard to how often and how long a patient's drug-induced delirium lasts. $\frac{58}{}$ The diagnosis is easily missed unless clinicians maintain a high index of suspicion. Indeed, three out of four patients experiencing delirium will be missed by healthcare professionals unless they use validated clinical tools. ${ }^{59-62}$ This is because the patients are usually experiencing a brain dysfunction that is called hypoactive or 'quiet' delirium, which is manifested by negative symptoms such as the inability to pay attention and disorganised thinking.

\section{Suggestions for improvement}

We recommend that the broad terminology brush that is quite often used in referring to various end-of-life practices be replaced with one that clearly defines each practice and explains how that particular practice is different than other practices. The refined, specific, and detailed brush will help in avoiding confusion and in explaining various options that are available in treating patients in the last stage of their lives. This issue is of utmost importance as language has significant effects on reality. Unclear terminology leads at best to confusion and at worst to abuse, obfuscation and disregard for the processes underpinning the ethical administration of sedation in the management of refractory suffering at the end of life.

The Belgian Advisory Committee on Bioethics should agree on the relevant definitions and adhere to them so all people, including scholars, physicians and nurses, know when they are speaking about the same practice. According to Broeckaert et al, 'palliative sedation is the intentional administration of sedative drugs in dosages and combinations required to reduce the consciousness of a terminal patient as much as necessary to adequately relieve one or more refractory 
symptoms'. ${ }_{3364}$ Chambaere et al, on the other hand, use a narrower and more general definition of palliative sedation in their survey, namely 'the use of one or more drugs to keep the patient deeply sedated or in a coma until death'. ${ }_{5}$ Chambaere et al's definition does not refer to the physician's intention and instead of reducing consciousness it speaks of 'coma until death.' All the qualifications in Broeckaert et al's definition that accentuate adequate and proportional palliative sedation in accordance with the patient's medical condition are missing from Chambaere et al's definition. This represents the sort of 'drift' in terminology that is potentially so hazardous.

The Belgian 'Palliative Sedation Directive' guidelines are of importance. ${ }^{66} \underline{67}$ Because sedation diminishes the patient's ability to interact with his or her surroundings, it should be done judiciously and carefully. This directive for the use of palliative sedation includes the presence of refractory physical, psychological and/or existential suffering; the drugs that should be used; the need for communication with patients and their families; and good control mechanisms. The directive explains the meaning of physical and psychological refractory symptoms. It instructs that the dosages and combinations to be administered should be proportional to the specific patient's condition, aiming to alleviate suffering; consultation with patient and relatives should be sought. The medical team should strive to have the best possible communication with patients and their families. When a patient is competent, the informed consent of the patient should be secured. Emphasis is put on monitoring, instructing to ensure that all relevant information relating to patients, their condition, the decision to administer palliative sedation, the

prescription for the implementation, evaluation and any adjustments to the dose are defined in the patients' medical files. Also, it is important to remember that if clinically appropriate and judged in the patient's best interest and in accordance to his/her preferences, palliative sedation can be reversed or stopped. Should clinical circumstances change qualitatively or quantitatively, the drugs can be stopped to allow the patient to communicate better with the loved one or clinical team.

Because this Palliative Sedation Directive is relatively new, it is likely that many medical professionals are unaware of its specifications. It is recommended to employ clear terminology by elucidating the differences between euthanasia and palliative sedation. The ethical dimensions of the practices (autonomy, consent, proportionality of treatment, goals of treatment) have direct, practical implications on the patient's treatment. Medical records should include detailed documentation relating to the medical condition, patient's wishes, discussions with the patient and/or her loved ones, treatment decisions including the use of palliation, the use of artificial hydration when appropriate and the effects of the intervention.

According to the Belgian Palliative Sedation Directive, sedation can be used for patients with a life expectancy of a few days up to 1 week. ${ }^{6667}$ The 2014 Deyaert et al study is disconcerting because it shows that physicians had shortened lives although they estimated that the patients could have lived longer than 1 week in 
$6.4 \%$ of the cases, and in almost $80 \%$ of cases they did not secure the patient's consent. ${ }^{23}$ Chambaere et al noted that in some cases alternatives to sedation had been possible for the treatment of symptoms and that continuous deep sedation would then be ethically questionable. ${ }^{56}$

Palliative sedation in the terminal stage of a patient's life requires a specialist's knowledge. Oftentimes, a critical care team consults an expert in palliative care when deciding to embark on such a path of treatment.iii ${ }^{68}$ Such a consultation can improve the quality of the decision-making process, provide insights that nonexperts may lack and is likely to serve the patient's best interests in providing the optimum care at the end of life. $\frac{667}{6}$

Broeckaert et al argue that the palliative care knowledge and expertise of the average physician is very limited. Most physicians have little training in palliative sedation or no training at all. Broeckaert et al maintain that at their own Catholic University of Leuven, the number of cases of palliative sedation had dropped from $7 \%$ in the first year of the palliative care unit (1999-2000) to an annual rate of 1\%$2 \%$ since 2001 . 99 patients and their loved ones should be counselled about the patient's condition, available options for treatment, contingency plans and implications of induced unconsciousness so as to enable them to make their preferred choice. Open discussions should be held with the medical team accentuating that sedation should not be used as a short cut, instead of providing comprehensive palliative care to the patient, including spiritual and emotional support. Belgian experts recommended that interdisciplinary team approaches, involving physicians, nurses, physiotherapists and pharmacists are needed to optimise sedative management. Unfortunately, they noted that these measures have not been widely incorporated into practice. $4^{\circ 0}$ More-interdisciplinary studies and work are required.

\section{Conclusions}

The differences between palliative sedation and euthanasia should be explained, clarified and elucidated, relating to patient's consent and autonomy, the performance of the two practices and the explicit legal directives for euthanasia. Open discussions should be held about the decision-making process and who should be involved in it. Physicians may also benefit from discussion on decision-making processes and the appropriate use of opioids and other medications that might shorten life.

The alarming and documented (though under-reported) problem of many thousands of patients being treated by physicians who intentionally hasten death without the patient's consent should be addressed. In 2007, the use of life-ending drugs with the intention to shorten life and without explicit request occurred in $1.8 \%$ of deaths ${ }^{70}$ and in 2013 it was $1.7 \%$ of deaths. ${ }^{22}{ }^{71}$ Sometimes itis clear from reports that there was no surrogate or proxy consent, and sometimes this issue is omitted. Since those in a terminal state or conditions of intractable suffering are frequently 
unable to consent, the issue is important. If proxy consent was garnered, it is a very different issue than if consent was not attempted or unsuccessfully obtained from the patient or his/her surrogate. Details should be provided and data meticulously tracked in this area.

Defenders of the Belgian policy and practices at the end of life argue that the Belgian model is 'a well-functioning experiment and probably a prototype of things to come elsewhere'. ${ }^{24}$ We disagree with regard to the first half of the statement and voice hope that the Belgian model will not become a prototype of things to come elsewhere. Supporters of the Belgian model argue that they have one of the best palliative care systems in the world, while research points, time and again, to severe deficiencies and abuses. Recent research regarding the use of quality indicators in palliative care shows that there are many barriers in the implementation of quality measurement such as lack of time and skills, the need for good leadership, the need for quality training and the need to learn from other services in the field. The researchers recommended the development of a process evaluation plan to assess the quality of the implementation process and the effectiveness of the palliative care strategies. Throughout the whole implementation process, involvement of stakeholders should be retained to evaluate critically the progression towards a systematic quality monitoring system of palliative care provided to patients. ${ }^{72}$

The Belgian attitude to end-of-life care has become too lax. Given that ending patients' lives without request is more common than euthanasia, ${ }_{13}$ and the significant number of palliative and/or terminal sedation cases, the need for equipping healthcare providers with adequate education and training is accentuated. As shown, research conducted in Belgium does not convince that its physicians have the awareness and knowledge of the different types of sedation and how they differ from euthanasia. The Belgians are researching the way their dying patients are being handled in a medical context. Their culture of selfsearching is certainly necessary. They need to develop the policy and means to assure that patients receive quality of care that serve the patients' best interests.

\section{Acknowledgments}

We thank the Editor and referees of BMJ Supportive and Palliative Care as well as Bert Broeckaert, Dan Callahan, Art Caplan, Nathan Cherny, Richard Collin, John Lantos, Annmarie Hosie, Tim Quill, Kasper Raus, Paul Schotsmans, and Jane Seymour for their constructive comments.

\section{References}

1. Smets T, Cohen J, Bilsen J, et al The labelling and reporting of euthanasia by Belgian physicians: a study of hypothetical cases. Eur J Public Health 2012;22:19-doi:10.1093/eurpub/ckq180

2. Papavasiliou EE, Payne S, Brearley S Current debates on end-of-life sedation: an international expert elicitation study. Support Care Cancer 2014;22:2141-9.doi:10.1007/s00520-014-2200-9

3. Douglas C, Kerridge I, Ankeny R Managing intentions: the end-of-life administration of analgesics and sedatives, and the possibility of slow euthanasia. Bioethics 2008;22:388- 96.doi:10.1111/j.1467-

8519.2008.00661.x

4. Quill TE The ambiguity of clinical intentions. N Engl J Med 1993;329:1039-

40.doi:10.1056/NEJM199309303291414

5. Douglas $C D$, Kerridge IH, Rainbird KJ, et al The intention to hasten death: a survey of attitudes and practices of surgeons in Australia. Med J Aust 2001;175:511-5. 
6. Rietjens JA, van der Heide $A$, Vrakking AM, et al Physician reports of terminal sedation without hydration or nutrition for patients nearing death in the Netherlands. Ann Intern Med 2004;141:178- 85.doi:10.7326/0003 4819-141-3-200408030-00006

7. Cherny NI, Radbruch L Board of the European Association for Palliative Care. European Association for Palliative Care (EAPC) recommended framework for the use of sedation in palliative care. Palliat Med 2009;23:581-93.doi:10.1177/0269216309107024

8. Bilsen J, Stichele RV, Mortier F, et al The incidence and characteristics of end-of-life decisions by GPs in Belgium. Fam Pract 2004;21:282-9.doi:10.1093/fampra/cmh312

9. Papavasiliou EE, Chambaere K, Deliens L, et al Physician-reported practices on continuous deep sedation until death: A descriptive and comparative study. Palliat Med 2014;28:491- 500.doi:10.1177/0269216314530768 10. Chambaere $\mathrm{K}$ Continuous deep sedation until death in different care settings in Belgium. Presentation given on 10 Jun 2010.

11. Vincent J-L . Carte blanche: "Maintenons la santé, mais pas la vie à tout prix". Le Soir 2015.

http://www.lesoir.be/478262/articl e/mai nten ant -sur-lesoirb e/2014-02- 25/carte-blanche-mainten ons-sant emais-p as-vie-tout-prix

12. Vincent JL, Schetz M, De Waele JJ, et al "Piece" of mind: end of life in the intensive care unit statement of the Belgian Society of Intensive Care Medicine. J Crit Care 2014;29:174- 5.doi:10.1016/j.jcrc.2013.08.025

13. Rietjens JA, van Delden JJ, van der Heide A, et al Terminal sedation and euthanasia: a comparison of clinical practices. Arch Intern Med 2006;166:749-53.doi:10.1001/archint e.166.7 .74 9

14. van der Heide A, Onwuteaka-Philipsen BD, Rurup ML, et al End-of-life practices in the Netherlands under the Euthanasia Act. N Engl J Med 2007;356:1957-65.doi:10.1056/NEJMs a071143

15. Materstvedt LJ, Magelssen M Medical murder in Belgium and the Netherlands. J Med Ethics 2016;42:6214.doi:10.1136/medethics-2015-103128

16. Lo B, Rubenfeld G Palliative Sedation in Dying Patients. JAMA 2005;294:1810-

6.doi:10.1001/jama.294.14.1810

17. Belgian Act on Euthanasia, Chapter II, Section 3, no. 1. http://www.ethical- perspectives.be/viewpi c.php? $L A N=E \& T A B L E=E P \& I D=59$

18. Belgian Act on Euthanasia, Chapter II, Section 3, no. 3(2). http://www.ethical- perspectives.be/viewpi c.php? $\mathrm{LAN}=\mathrm{E} \& T A \mathrm{BLE}=\mathrm{EP} \& \mathrm{I} \mathrm{D}=59$

19. Smets T, Bilsen J, Cohen J, et al. Legal euthanasia in Belgium: characteristics of all reported euthanasia cases. Med Care 2010;48:187-92.doi:10.1097/MLR.obo13e3181bd4dd e

20. Bilsen J, Cohen J, Chambaere K, et al Medical end-of-life practices under the euthanasia law in Belgium. N Engl J Med 2009;361:1119-21.doi:10.1056/NEJMcog04292

21. Rise in assisted deaths Presseurop. 2011 http://www.presseurop.eu/en/cont ent/n ews -bri ef- cover/878961rise-assisted-deaths

22. Chambaere $K$, Vander Stichele $R$, Mortier $F$, et al Recent trends in euthanasia and other end-of-life practices in Belgium. N Engl J Med 2015;372:1179-81.doi:10.1056/NEJMc1414527

23. Deyaert J, Chambaere K, Cohen J, et al Labelling of end-of-life decisions by physicians. J Med Ethics 2014;40:505-7.doi:10.1136/medethics-2013-101854

24. Bernheim JL, Distelmans W, Mullie A, et al Questions and answers on the Belgian model of integral end-oflife care: experiment? prototype? J Bioeth Inq 2014;11:507-29.doi:10.1007/s11673-014- 9554-Z

25. Bernheim JL, Chambaere K, Theuns $P$, et al State of palliative care development in European Countries with and without legally regulated physician-assisted dying. Health Care 2014;2:10-14.doi:10.12966/hc.02.02.2014 26. Raus K, Anquinet L, Rietjens J, et al Factors that facilitate or constrain the use of continuous sedation at the end of life by physicians and nurses in Belgium: results from a focus group study. J Med Ethics 2014;40:2304.doi:10.1136/medethics-2012-100571

27. Sterckx S, Raus K, Mortier F, eds. Continuous sedation at the end of life - clinical, legal and ethical perspectives. Cambridge: Cambridge University Press, 2013.

28. Seymour J, Rietjens J, Brown J, et al The perspectives of clinical staff and bereaved informal care-givers on the use of continuous sedation until death for cancer patients: the study protocol of the UNBIASED study. BMC Palliat Care 2011;10:5.doi:10.1186/1472-684X-10-5

29. Raus K, Sterckx S, Mortier F Continuous deep sedation at the end of life and the 'natural death' hypothesis. Bioethics 2012;26:329-36.doi:10.1111/j.1467-8519.2010.01861.x

30. Robijn L, Chambaere K, Raus K, et al Reasons for continuous sedation until death in cancer patients: a qualitative interview study. Eur J Cancer Care 2017;26:e12405.doi:10.1111/ecc.12405

31. Rys $S$, Mortier F, Deliens $L$, et al The practice of continuous sedation until death in nursing homes in Flanders, Belgium: a nationwide study. J Am Geriatr Soc 2014;62:1869-76.doi:10.1111/jgs.13073

32. Broeckaert B Palliative sedation, physician-assisted suicide, and euthanasia: "same, same but different"? Am J Bioeth 2011;11:62-4.doi:10.1080/15265161.2011.577518

33.Kelley AS, Meier DE Palliative care - a shifting paradigm. N Engl J Med 2010;363:781- 
2.doi:10.1056/NEJMe1004139

34. Pastrana $T$, Jünger $S$, Ostgathe $C$, et al $A$ matter of definition - key elements identified in a discourse analysis of definitions of palliative care. Palliat Med 2008;22:222-32.doi:10.1177/0269216308089803

35. de Graeff A, Dean M Palliative sedation therapy in the last weeks of life: a literature review and recommendations for standards. J Palliat Med 2007;10:67-85.doi:10.1089/jpm.2006.0139

36. Olsen ML, Swetz KM, Mueller PS Ethical decision making with end-of-life care: palliative sedation and withholding or withdrawing life-sustaining treatments. Mayo Clin Proc 2010;85:949-54.doi:10.4065/mcp.2010 .0201

37. Bruinsma SM, Rietjens JA, Swart SJ, et al Estimating the potential life-shortening effect of continuous sedation until death: a comparison between two approaches. J Med Ethics 2014;40:458-

62.doi:10.1136/medethics-2013-101459

38. Cherny NI, Radbruch L The Board of the European Association for Palliative Care. European Association for Palliative Care (EAPC) recommended framework for the use of sedation in palliative care. Palliat Med 2009;23:581- 93.doi:10.1177/0269216309107024

39. Cherny NI Sedation for the care of patients with advanced cancer. Nat Clin Pract Oncol 2006;3:492500.doi:10.1038/ncponc0583

40. Salgado DR, Favory R, Goulart M, et al Toward less sedation in the intensive care unit: a prospective observational study. J Crit Care 2011;26:113-21.doi:10.1016/j.jcrc.2010.11.003

41. Beller EM, van Driel ML, McGregor L, et al Palliative pharmacological sedation for terminally ill adults. Cochrane Database Syst Rev 2015;1:CD010206.doi:10.1002/14651858.CD010206.pub2

42. Maltoni M, Scarpi E, Rosati M, et al Palliative sedation in end-of-life care and survival: a systematic review. J Clin Oncol 2012;30:1378-83.doi:10.1200/JCO.2011.37.3795

43. Maltoni M, Pittureri C, Scarpi E, et al Palliative sedation therapy does not hasten death: results from a prospective multicenter study. Ann Oncol 2009;20:1163-9.doi:10.1093/annonc/mdpo48

44. Bakker J, Jansen TC, Lima A, et al Why opioids and sedatives may prolong life rather than hasten death after ventilator withdrawal in critically ill patients. Am J Hosp Palliat Care 2008;25:152-

4.doi:10.1177/1049909108315511

45.Temel JS, Greer JA, Muzikansky A, et al Early palliative care for patients with metastatic non-small-cell lung cancer. N Engl J Med 2010;363:733-42.doi:10.1056/NEJMo a1000678

46. Anquinet L, Raus K, Sterckx S, et al Similarities and differences between continuous sedation until death and euthanasia - professional caregivers' attitudes and experiences: a focus group study. Palliat Med 2013;27:55361.doi:10.1177/0269216312462272

47. Jones AD, Gastmans C, MacKellar C, Sterckx S, Raus K The practice of continuous sedation at the end of life in Belgium. In: Jones AD, Gastmans C, MacKellar C, eds. Euthanasia and assisted suicide: lessons from Belgium. Cambridge: Cambridge University Press, 2017:86-100.

48. Enck RE Drug-induced terminal sedation for symptom control. Am J Hosp Palliat Care 1991;8:35.doi:10.1177/104990919100800501

49. Ten Have $H$, Clarke D Broeckaert B, Nuñez Olarte JM Sedation in palliative care. Facts and concepts. In: Ten Have $\mathrm{H}, \mathrm{ClarkeD}$, eds. The Ethics of Palliative Care. European Perspectives. Buckingham: Open University Press, 2002:166-80

50. Chater S, Viola R, Paterson J, et al Sedation for intractable distress in the dying - a survey of experts. Palliat Med 1998;12:255-69.doi:10.1191/026921698671831786

51. Carver AC, Foley KM The Wein article reviewed. Oncology 2000;14:597-8.

52. Krakauer EL Responding to intractable terminal suffering. Ann Intern Med 2000;133:56o.doi:10.7326/00034819-133-7-200010030-00020

53.Sulmasy DP, Ury WA, Ahronheim JC, et al Responding to intractable terminal suffering. Ann Intern Med 2000;133:560-1.doi:10.7326/0003-4819-133-7-200010030-00021

54. Miccinesi G, Rietjens JA, Deliens L, et al Continuous deep sedation: physicians' experiences in six European countries. J Pain Symptom Manage 2006;31:122-9.doi:10.1016/j.jpainsym man .2005.0 7.004

55. Claessens $P$, Menten J, Schotsmans $P$, et al Palliative sedation: a review of the research literature. J Pain Symptom Manage 2008;36:310-33.doi:10.1016/j.jpainsymman .2007.10.004

56. Chambaere K, Bilsen J, Cohen J, et al Continuous deep sedation until death in Belgium: a nationwide survey. Arch Intern Med 2010;170:490-3.doi:10.1001/archinternmed .2009.542

57 .

Anquinet $L$, Rietjens JAC, Seale $C$, et al The practice of continuous deep sedation until death in flanders

(belgium), the Netherlands, and the U.K: a comparative study. J Pain Symptom Manage 2012;44:33-

43.doi:10.1016/j.jpainsymman.2011.07.007

58. Moore AR, O'Keeffe ST Drug-induced cognitive impairment in the elderly. Drugs Aging 1999;15:15-

28.doi:10.2165/00002512-199915010-00002

59. Inouye SK, Foreman MD, Mion LC, et al Nurses' recognition of delirium and its symptoms: comparison of 
nurse and researcher ratings. Arch Intern Med 2001;161:2467-73.

6o. Devlin JW, Fong JJ, Schumaker G, et al Use of a validated delirium assessment tool improves the ability of physicians to identify delirium in medical intensive care unit patients. Crit Care

Med 2007;35:2721-4.doi:10.1097/00003246-200712000-00007

61. Spronk PE, Riekerk B, Hofhuis J, et al Occurrence of delirium is severely underestimated in the ICU during daily care. Intensive Care Med 2009;35:1276-80.doi:10.1007/s00134-009-1466-8

62. van Eijk MM, van Marum RJ, Klijn IA, et al Comparison of delirium assessment tools in a mixed intensive care unit. Crit Care Med 2009;37:1881-5.doi:10.1097/CCM.obo13e3181a00118

63. Claessens P, Menten J, Schotsmans P, et al Palliative sedation, not slow euthanasia: a prospective, longitudinal study of sedation in Flemish palliative care units. J Pain Symptom Manage 2011;41:14-

24.doi:10.1016/j.jpainsymman.2010.04.019

64 Broeckaert B Palliative sedation defined or why and when terminal sedation is not euthanasia. J Pain Symptom Manage 2000;20:S58.

65. Chambaere K, Rietjens JA, Cohen J, et al Palliative sedation in Flemish palliative care units. J Pain Symptom Manage 2011;41:e1-e2.doi:10.1016/j.jpainsym man .2011.03.005

66. Broeckaert B, Mullie A, Gielen J, et al Palliatieve Sedatie, Richtlijn, Versie:2.0. 2012

http://www.pallialine.be/accounts/129/docs/richtlijn_palliatieve_sedatie.pdf.

67. Broeckaert B, Mullie A, Gielen J, et al Palliative sedation directive, Version: 2.0. (June 30, 2012, in English).

68. Royal Dutch Medical Association (KNMG). Guideline for palliative sedation. Utrecht: Committee on National Guideline for Palliative Sedation, 2009:68.

69. Broeckaert B, Claessens $P$, Schotsmans $P$, et al What's in a name? Palliative sedation in Belgium. Reply to Chambaere et al. J Pain \& Symptom Management 2011;41:e3

70. Chambaere K, Bilsen J, Cohen J, et al Physician-assisted deaths under the euthanasia law in Belgium: a population- based survey. Can Med Assoc J 2010;182:895-901.doi:10.1503/cmaj.091876

71. Cohen-Almagor R First do no harm: intentionally shortening lives of patients without their explicit request in Belgium. J Med Ethics 2015;41:625-9.doi:10.1136/medethics-2014-102387

72. Leemans $K$, Van den Block $L$, Vander Stichele $R$, et al How to implement quality indicators successfully in palliative care services: perceptions of team members about facilitators of and barriers to implementation.

Support Care Cancer 2015;23:3503-11.doi:10.1007/s00520-015-2687-8

73. van der Heide A, Deliens L, Faisst K, et al End-of-life decision-making in six European countries: descriptive study. Lancet 2003;362:345-50.doi:10.1016/S0140-6736(03)14019-6 